\title{
Coherent Control of Polariton Parametric Scattering in Semiconductor Microcavities
}

\author{
S. Kundermann, M. Saba, C. Ciuti, ${ }^{*}$ T. Guillet, U. Oesterle, J. L. Staehli, and B. Deveaud \\ Institut de Photonique et d'Electronique Quantiques, Ecole Polytechnique Fédérale de Lausanne (EPFL), \\ CH-1015 Lausanne, Switzerland \\ (Received 23 August 2002)
}

\begin{abstract}
In a pump-probe experiment, we have been able to control, with phase-locked probe pulses, the ultrafast nonlinear optical emission of a semiconductor microcavity, arising from polariton parametric amplification. This evidences the coherence of the polariton population near $k=0$, even for delays much longer than the pulse width. The control of a large population at $k=0$ is possible although the probe pulses are much weaker than the large polarization they control. With rising pump power the dynamics of the scattering get faster. Just above threshold the parametric scattering process shows unexpected long coherence times, whereas when pump power is risen the contrast decays due to a significant pump reservoir depletion. The weak pulses at normal incidence control the whole angular emission pattern of the microcavity.
\end{abstract}

DOI:

In the past few years, microcavities working in the strong coupling regime have attracted quite a lot of attention [1-3]. The excitonic transition of the embedded quantum well is strongly coupled to the cavity photon mode. In the radiative region near $k=0$ the resonant exciton and photon modes split and give rise to composite bosons, the so-called microcavity polaritons [4]. The dispersion of the lower polariton (LP) strongly deviates from the unperturbed exciton dispersion. The particular shape of the lower polariton dispersion allows for a parametric polariton scattering process conserving energy and in-plane momentum $[2,5,6]$. Two polaritons with an in-plane momentum $k_{p}$ scatter into a signal-idler pair with zero and $2 k_{p}$ momenta. The microcavity can thus be understood as an optical parametric oscillator.

It is well-known that the parametric oscillation in a classical optical parametric oscillator (OPO) is a fully coherent process. The crystal is pumped in the transparency region and the coherence time of the process is given by the duration of the pump pulses. The pump intensity required to achieve parametric oscillation is very high because the involved electronic states are virtual $[7,8]$. The semiconductor microcavity system exhibits three major differences to the classical OPO. First, the signal, pump, and idler states are real and thus very efficiently coupled to external laser light. Second, the excitations are interacting via a real Coulomb interaction which results in high parametric scattering rates. These two features illustrate the high efficiency of the process $[2,5,6]$. The third difference is that for our system the coherence time should not be given by the external laser pulses but by the properties of the excitations and the scattering themselves. The coherent control technique allows one to sense these coherence properties and to manipulate the scattering within its coherence time [9-11].

In this Letter we report the coherent control of the parametric polariton scattering. The dynamics of the parametric scattering are governed by the lifetime of
PACS numbers: 78.47.+p, 05.30.Jp, 42.65.-k, 78.67.-n

the real polariton states and the applied pump power [12]. Especially just above threshold the dynamics of the polariton scattering are very slow. In fact, in our high-quality microcavity we demonstrate that the scattering can be coherently controlled during the whole lower polariton lifetime which is in our sample of the order of 10 ps. Comparing again with the classical OPO, in our cavity the coherent control is possible over a delay of about 10 ps which is almost 100 times longer than the laser pulse duration. The comparison between single probe and coherent control measurements shows that under moderate excitation density the signal polaritons interfere and the total (time integrated) amplified signal can be considered as the result of an independent superposition of two signal polariton emissions arising from the respective two probe pulses. For very high pump power, however, the pump polariton reservoir is considerably depleted and the independence is no more given. Furthermore, we have measured the complete angular emission pattern. It is shown that the emissions from signal and idler states are maximized for constructive probe interference and that the pump reservoir can be depleted due to the scattering.

The sample we investigated is a high-quality GaAs $\lambda$ cavity with a single InGaAs quantum well inside (see Fig. 1 and Ref. [13] for details). The minimum polariton splitting is $3.6 \mathrm{meV}$ and the FWHM polariton spectral linewidth is $\simeq 0.1 \mathrm{meV}$ which corresponds to a polariton lifetime of about $10 \mathrm{ps}$. The sample is cooled down to about $10 \mathrm{~K}$ in a cold finger cryostat with wide optical aperture. The excitation pulses are emitted by a modelocked Ti:sapphire laser with an $80 \mathrm{MHz}$ repetition rate. The pump pulses, after passing a spectral filter, have a $\simeq 2 \mathrm{meV}$ spectral width (FWHM, corresponding to a $\simeq 1$ ps duration) and are tuned to the polariton energy at the excitation angle. The probe pulses are $150 \mathrm{fs}$ long ( $15 \mathrm{meV}$ spectral linewidth) and hit the sample at normal incidence. The phase-locked delay between the 


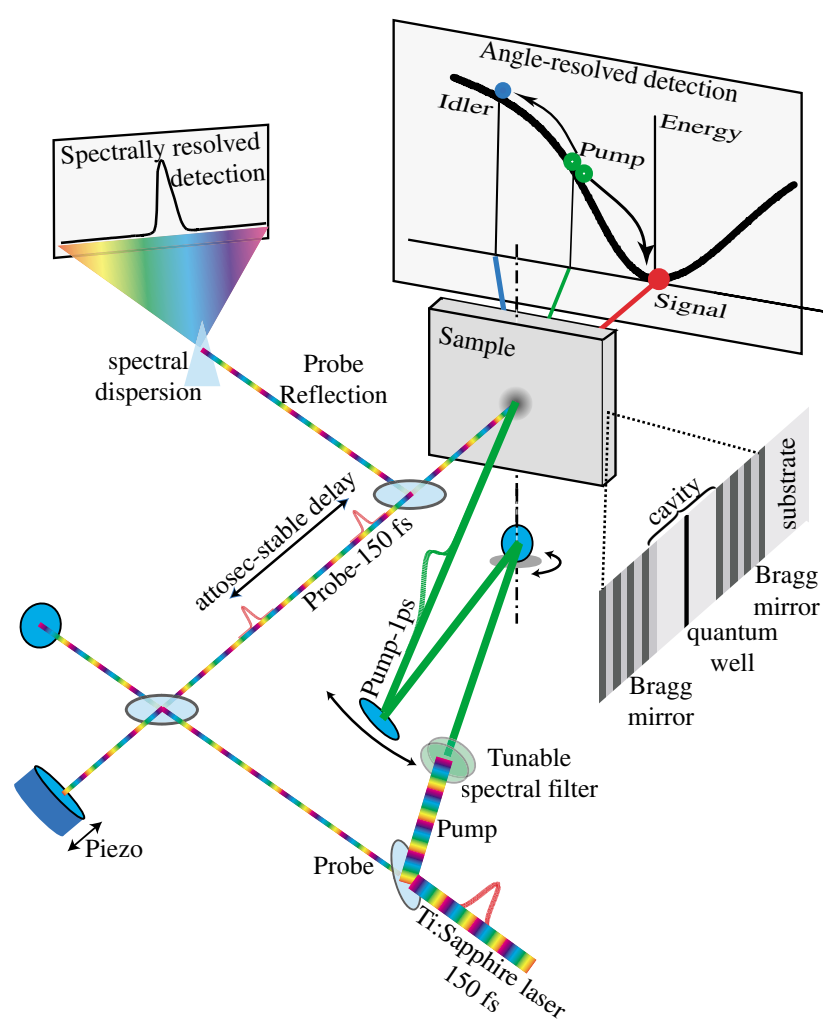

FIG. 1 (color). Experimental setup: the pump (spectrally narrow and temporally long) hits the sample with a variable angle $\left(\simeq 10^{\circ}\right.$ in the experiments presented here), while the two probe pulses (spectrally broad and temporally short) are in the normal direction. The reflected or transmitted probe, which is greatly amplified by the parametric scattering, is spectrally dispersed and detected. The angular pattern of the emitted light is also detected. The parametric scattering process is sketched along the polariton dispersion curve. The sample structure is also represented, with the cavity $\lambda$ spacer delimited by the two Bragg mirrors and the quantum well at the cavity center.

two probe pulses is generated in an actively stabilized Michelson interferometer [14] (see Fig. 1). The delay between the pulses is stable within $10 \mathrm{~nm}$ which corresponds to a phase stability at the polariton wavelength of $\simeq \pi / 40$. The reflection and the transmission of the probe beam are detected with a charge-coupled device (CCD) camera after spectral dispersion in a monochromator. The emission on the substrate side is also collected by a lens and can be imaged on a CCD in order to measure the angular emission pattern.

In Figs. 2(a) and 2(b) the parametric scattering process in a pump and single probe experiment is shown. Figure 2(a) shows the probe transmission spectrum. Without pump, we observe two transmission peaks at the lower and upper polariton energy (dashed line). The lower polariton peak is 10 times more intense than the upper one since we are at negative detuning between the photon and the exciton resonance of $1 \mathrm{meV}$ (the measured linewidth is $\simeq 0.3 \mathrm{meV}$, the spectral resolution

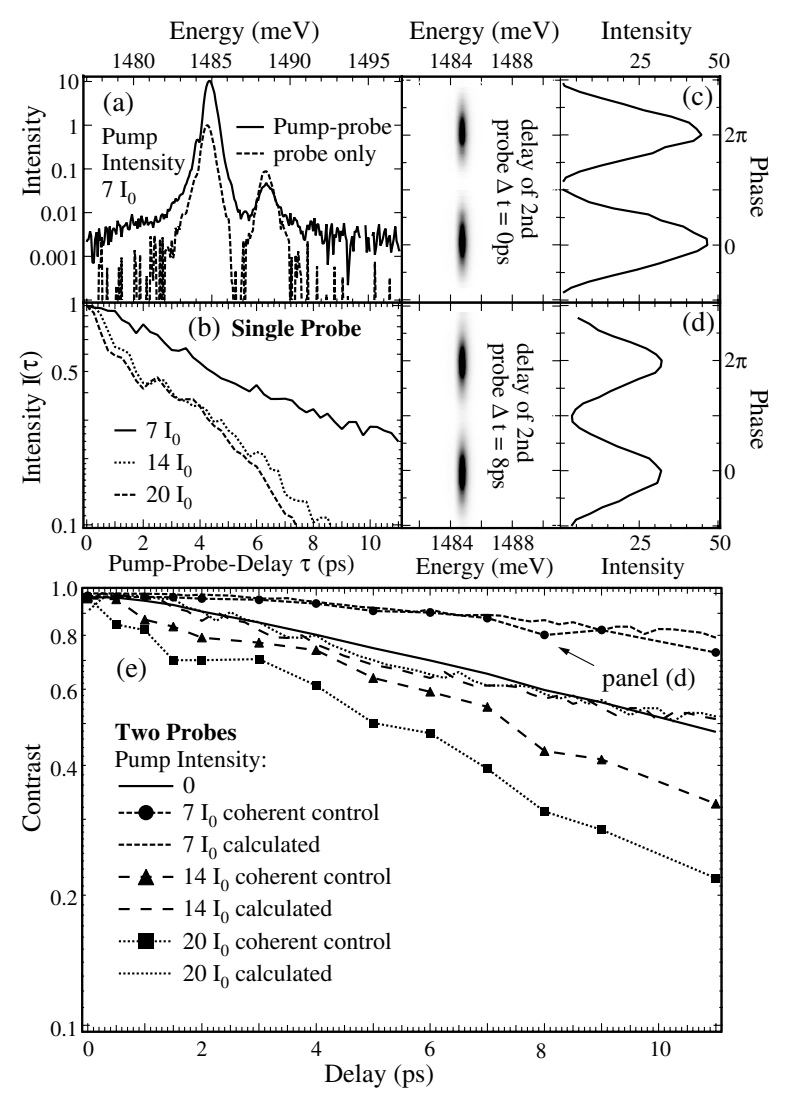

FIG. 2. (a) Probe-transmission spectra with (full line) and without (dashed line) pump excitation; one probe pulse only hits the sample. At the chosen position on the sample, the energy of the empty-cavity mode $(1486.0 \mathrm{meV})$ is slightly lower than the bare exciton energy (1487.0 meV). (b) Evolution of the time integrated signal emission as the probe to pump delay is scanned. The intensity has been integrated over the whole peak width of the LP emission. (c),(d) Transmission spectra as a function of relative phase (control phase) between the two phase-controlled probe pulses. On the right the value of the intensity [integrated over the peak width as in (b)] is extracted. The signal is normalized with respect to the intensity of a single probe pulse and the pump luminescence has been subtracted. The second probe pulse is delayed with respect to the first one by $\Delta t=0$ (c), 8 ps (d). (e) Contrast of coherent control oscillations versus the delay between the two probe pulses; the first probe pulse is synchronous with the pump. If $I_{\max }$ and $I_{\min }$ are the maximum and minimum values observed in the coherent-control oscillations, the contrast $C$ is defined as $C=\left(I_{\max }-I_{\min }\right) /\left(I_{\max }+I_{\min }\right)$. The incident pump polariton densities are normalized to $I_{0}=9 \times$ $10^{9}$ polaritons $/\left(\mathrm{cm}^{2}\right.$ pulse $)$ which is the density at which the stimulation threshold is reached, whereas the density of a single probe is $10^{6}$ polaritons $/\left(\mathrm{cm}^{2}\right.$ pulse $)$. Each curve is measured for a different pump density, as indicated in the legend. The calculated contrast has been obtained from the experimental data shown in panel (b) as explained in the text.

of the detection apparatus). When the pump beam is switched on and tuned at $\mathrm{a} \simeq 10^{\circ}$ incidence angle, the probe transmission is greatly amplified. The spectral position of the gain peak (slightly shifted with respect to 
the unperturbed lower polariton energy), the pump angle for maximum efficiency $\left(\simeq 10^{\circ}\right)$ and the threshold on the pump power (not shown) are in agreement with the model of the polariton parametric amplifier [12].

In panel 2(b) the normalized signal emission at $k=0$ is depicted versus pump-probe delay. The decay of the signal is not monoexponential. The decay is faster for higher pump power and the $14 I_{0}$ and $20 I_{0}$ curves show similar behavior.

Panels 2(c) and 2(d) show the emission intensity of the signal states for the coherent control experiment. The pump and the first probe hit the sample simultaneously, whereas the second probe is delayed and phase locked by the Michelson interferometer. The evolution of the time integrated emission intensity of the signal is depicted as the phase between the probe pulses is scanned. At zero delay, when the two probes perfectly overlap, the coherent control is complete and the emission goes to zero for destructive interference of the probes. This is due to optical interference of the probes before reaching the cavity. When the second probe is delayed by 8 ps the oscillations almost go down to zero and the contrast is still around $80 \%$. The phase imprinted onto the polaritons by the first probe is conserved until the second probe enters the cavity and until the scattering process is completed.

To map out the coherence properties in detail the coherent control experiment has been performed for various delays and pump powers. The coherence time of the lower polariton at $k=0$ has been determined using only the two probe pulses in the coherent control configuration without the pump. The decay time extracted from Fig. 2(e) is 10 ps. When the pump is switched on and the power is adjusted to approximately 7 times the threshold intensity $I_{0}$ for the scattering, the contrast decays even more slowly than for the probes alone, evidencing a long lasting coherence for the polaritons [15].

In order to quantify the coherence degree and to estimate the influence of nonradiative dephasing we now compare coherent control experiments, sensitive to the coherence, and single probe experiments sensitive to the dynamics of the scattering. We calculate the interference contrast as if the amplification dynamics of the two probes were completely independent [16]. This is shown in Fig. 2(e) together with the measured contrast.

For the contrast at $I=7 I_{0}$ we observe that the measured and calculated contrast curves overlap, and therefore in this low pump power regime the coherent control experiment can be understood in terms of two independent scattering processes interfering with each other. This behavior occurs only if we assume that the signal polaritons have conserved their phase until the second probe arrives; i.e., the dynamics of the polaritons are purely coherent and limited by the radiative dephasing.

When pump power is risen to $14 I_{0}$ and $20 I_{0}$ the measured coherent control contrast presents a fast initial decay and deviates from the calculated contrast curve. The calculated corresponding contrast is, however, fixed near the values of the bare lower polariton contrast. It shows that for a pump power far above threshold the scattering processes are no more independent. This can be interpreted in terms of excitation induced dephasing [17] or in a depletion of the initial pump reservoir. Indeed the scattering rate into the signal and idler states is proportional to the square of the pump density [12], and the amplification of the first probe depletes the pump polariton supply.

For energy and momentum conservation, the parametric process has to involve idler polaritons [12], two polaritons from the pump being scattered into a signalidler pair. The effects of the coherent control should therefore appear all over the polariton dispersion and not only in the normal direction, where the probe pulses are detected. Figure 3 shows the angular pattern of the emission of the microcavity on the substrate side, opposite to the excitation side (all the spectrally integrated emission is collected). The measurements are taken in the coherent control configuration, as a function of the relative phase between the two pulses. The pump power was approximately the same as for $I=14 I_{0}$ in
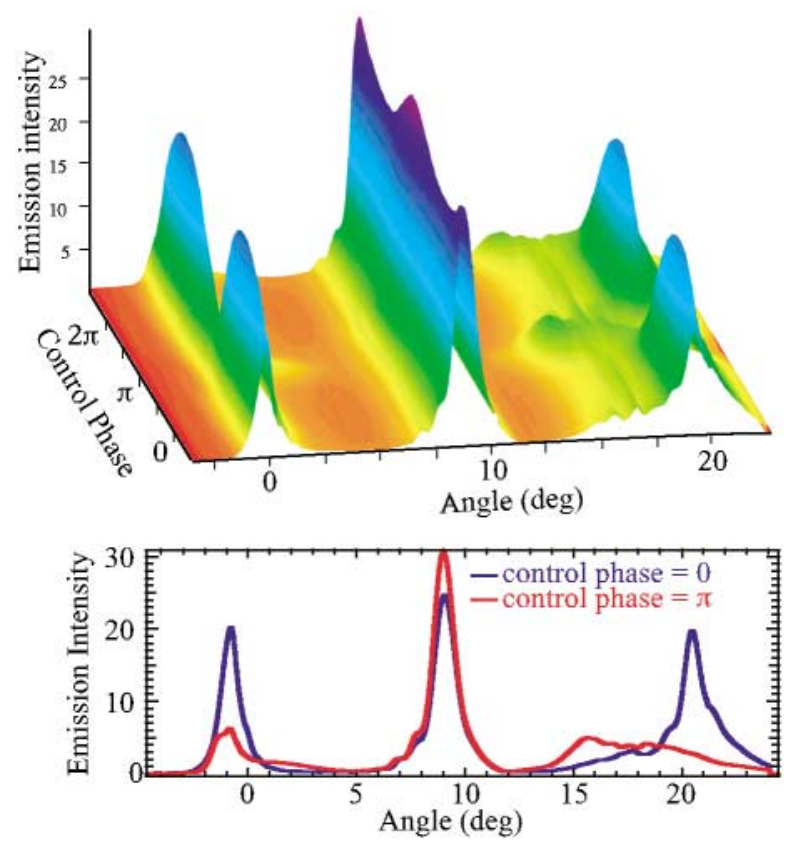

FIG. 3 (color). Angular pattern of the emission in transmission geometry as a function of the control phase. The delay between the two phase-locked probe pulses is 2 ps. The lower panel shows two sections of the surface plot corresponding to a constructive and a destructive control phase. The signal and pump emissions were attenuated (up to $14^{\circ}$ ) by 4 orders of magnitude with a neutral density filter in order to show the whole angular pattern in the same picture. To evidence the effects of the coherent control on the pump beam, the probe density employed here was higher $\left[2.5 \times 10^{8}\right.$ polaritons $/\left(\mathrm{cm}^{2}\right.$ pulse $\left.)\right]$ than that in the measurements in Fig. 2, while the pump density was lower $\left[\simeq 10^{11}\right.$ polaritons $/\left(\mathrm{cm}^{2}\right.$ pulse $\left.)\right]$. 
Fig. 2(e). The coarse delay between the two phase-locked probe pulses is $2 \mathrm{ps}$. The incidence angle $\theta$ is measured with respect to the normal direction, so that the probe is transmitted around zero angle, while the pump is at $\theta \simeq 9^{\circ}$ The idler branch is also visible at an angle slightly larger than twice the pump angle. This is because the parametric scattering conserves the in-plane momentum of polaritons, which is proportional to $\sin \theta$. Two sections of the surface plot are also shown to quantitatively compare the angular patterns obtained when the two probe pulses are in phase or out of phase. Signal and idler oscillations are in phase; i.e., when the coherent control maximizes the emission in the probe direction, the idler also is maximized. Emission from the idler states is thus correlated to the signal emission. Simultaneously, the antiphase with the pump beam is clearly seen in Fig. 3, as, for energy conservation, the polaritons which are not found in signal and idler branches have to be at the pump angle.

When the two probe pulses are in antiphase, the emission in the probe direction is strongly reduced and the polaritons at the pump angle decay spontaneously, as in the absence of an external seed, when the parametric scattering can be started by the few pump polaritons that relax into the band bottom, generating an emission around the normal direction $[6,18-20]$. For the measurements in Fig. 3, the incidence angles of pump $\left(\simeq 9^{\circ}\right.$, instead of the optimal $\simeq 10^{\circ}$ of Fig. 2$)$ and probe $\left(\simeq-1^{\circ}\right)$ have been chosen in order to distinguish the emission stimulated by the probe (occurring at $\simeq-1^{\circ}$ ) from that occurring at $\simeq 1.5^{\circ}$ after the "spontaneous" parametric scattering of the pump polaritons. The spontaneous parametric emission of the pump has a broad angular pattern and is correlated to an idler emission at $\simeq 17.5^{\circ}$. Remarkably, when the second probe interferes constructively, the emission generated by the pump alone is greatly reduced. This directly confirms the depletion of the pump reservoir at $I=14 I_{0}$, which has been assumed in the interpretation of Fig. 2(e).

In conclusion, we have demonstrated the coherent control of the polariton parametric scattering in a semiconductor microcavity. Thanks to the coherence and the stimulation effects in the parametric scattering of polaritons, weak subpicosecond laser pulses control a very intense optical emission. It is proven that the dynamics of the polaritons at $k=0$ are completely coherent at moderate pump power. The parametric scattering involves polaritons with different wave vectors, so that the control pulse hitting the sample at normal incidence is able to control streams of photons emitted along different directions. Polaritons are therefore a model system for studying Coulomb quantum kinetics, as they combine a very efficient interaction, needed for fast manipulation, with a low decoherence.

We thank G. Bongiovanni, M. A. Dupertuis, W. Elsäßer, J. D. Ganière, G. R. Hayes, R. Houdré, A. Mura, P. Schwendimann, and A. Quattropani for helpful discussions. Financial support from the Swiss National Science Foundation is acknowledged.

*Present address: Laboratoire de Physique de la Matière Condensée, 24, rue Lhomond, 75230 Paris Cedex 05, France.

[1] Y. Yamamoto, Nature (London) 405, 629 (2000).

[2] P. G. Savvidis et al., Phys. Rev. Lett. 84, 1547 (2000).

[3] G. Messin et al., Phys. Rev. Lett. 87, 127403 (2001).

[4] C. Weisbuch, M. Nishioka, A. Ishikawa, and Y. Arakawa, Phys. Rev. Lett. 69, 3314 (1992).

[5] M. Saba et al., Nature (London) 414, 731 (2001).

[6] R. M. Stevenson et al., Phys. Rev. Lett. 85, 3680 (2000).

[7] W. Brunner and H. Paul, Prog. Opt. 15, 3 (1977).

[8] V. G. Dmitriev, G. G. Gurzadyan, and D. N. Nikogosyan, Handbook of Nonlinear Optical Crystals, Springer Series in Optical Sciences Vol. 64 (Springer, Berlin, 1999).

[9] A. P. Heberle, J. J. Baumberg, and K. Köhler, Phys. Rev. Lett. 75, 2598 (1995).

[10] X. Marie et al., Phys. Rev. B 59, R2494 (1999).

[11] Y.-S. Lee et al., Appl. Phys. Lett. 78, 3941 (2001).

[12] C. Ciuti, P. Schwendimann, B. Deveaud, and A. Quattropani, Phys. Rev. B 62, R4825 (2000).

[13] M. Saba et al., Phys. Rev. Lett. 85, 385 (2000).

[14] M. U. Wehner, M. H. Ulm, and M. Wegener, Opt. Lett. 22, 1455 (1997).

[15] L. A. Dunbar et al., Phys. Rev. B 66, 195307 (2002).

[16] We evaluate the mean amplitude $A(\tau)$ of the polarization at $k=0$ as the square root of the time integrated signal intensity $I(\tau)$ in a single probe experiment. The calculated contrast is then equal to $C(\tau)=\left[|A(0)+A(\tau)|^{2}-\right.$ $\left.|A(0)-A(\tau)|^{2}\right] /\left[|A(0)+A(\tau)|^{2}+|A(0)-A(\tau)|^{2}\right]$.

[17] D.S. Chemla and J. Shah, Nature (London) 411, 549 (2001).

[18] J. J. Baumberg et al., Phys. Rev. B 62, R16 247 (2000).

[19] P. G. Savvidis et al., Phys. Rev. B 62, R13 278 (2000).

[20] C. Ciuti, P. Schwendimann, and A. Quattropani, Phys. Rev. B 63, 041303(R) (2001). 